\title{
Safety Mechanism for SkyTran Tracks
}

\author{
Yair Wiseman \\ Computer Science Department, Bar-Ilan University, Ramat-Gan 52900, Israel \\ wiseman@cs.biu.ac.il
}

\begin{abstract}
SkyTran track must be incessantly supervised in order to prevent fatal crashes that can be happened due to damaged tracks. We suggest a safety tool for an incessant inspection of SkyTran tracks by employing an inexpensive digital camera generating JPEG pictures. An automatic analysis can indicates whether a track segment is damaged and if a damage track segment is detected by the automatic analysis, the traffic in this segment will be halted straightaway in order to verify whether the track segment is indeed damaged and accordingly to fix the track segment if needed.
\end{abstract}

Keywords: SkyTran, Discrete Cosine Transform, JPEG

\section{Introduction}

IAI (Israel Aerospace Industries) in Israel and NASA (National Aeronautics and Space Administration) in USA founded the SkyTran [1,2] as a next generation Maglev transportation system [3]. Maglev is an acronym of Magnetic Levitation employed in a small number of transportion equipment and SkyTran will be one of them [4].

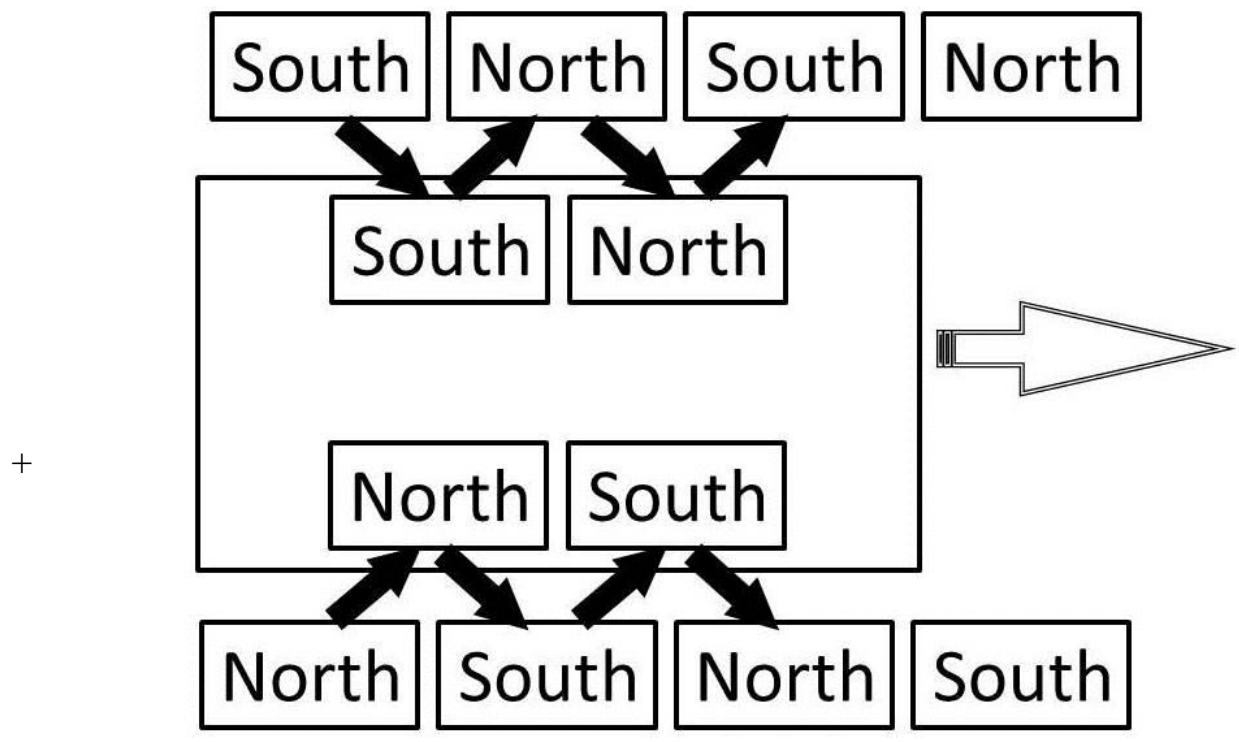

Figure 1. The Way Magnets Push and Pull Maglev Vehicle

Maglev vehicles have superconducting magnets on them and wire coils all along the tracks [5]. When the Maglev vehicle starts it will roll on rubber wheels; however as soon as the vehicle gets to about $90-100 \mathrm{mph}$, the superconducting magnets will generate magnetic field induction that will levitate the vehicle about 4-5 inches above the track, so except of starting off, Maglev vehicles do not make use of wheels, as a result they make less noise $[6,7]$. 
The magnets in the tracks are magnetized "North" and "South" alternately. Similarly, the magnets in the vehicles are also magnetized "North" and "South" alternately. The magnets pull and push the vehicle forward as shown in Figure 1. Since the coils on the two sides of the tracks are connected, the induced current will stabilize the train in a case of an unpredictable unbalanced ride [8].

SkyTran will make use of magnetic levitation to move compact vehicles without making contact with its tracks; nor with the ground. The compact vehicles will go along tracks installed at a height of several meters. The vehicles will make use of magnets in order to create both forward motion and avoiding contact with its tracks, so as to prevent friction. The SkyTran vehicle will have an aerodynamic shape. A model of the vehicle can be seen in Figure 2.

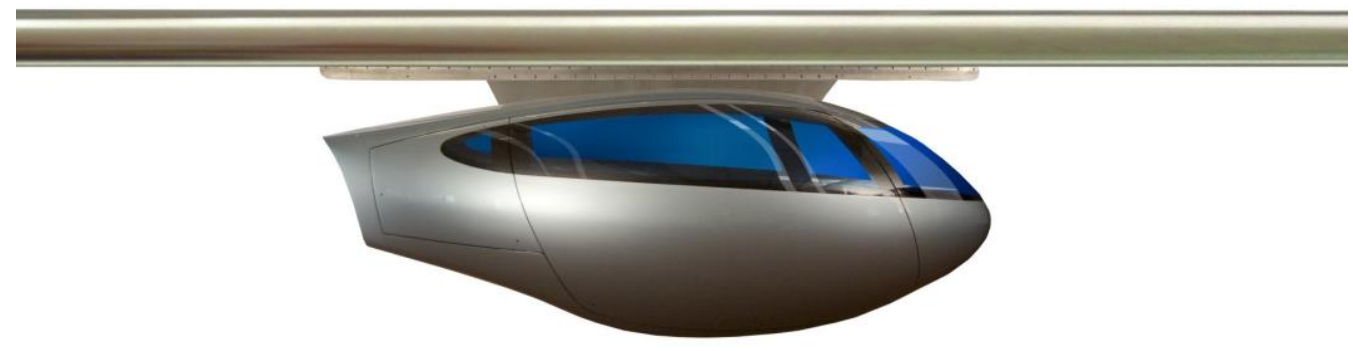

Figure 2. SkyTran Vehicle and Track

SkyTran vehicles will not contain any moving parts like wheels. As a result, they will travel more quietly and more smoothly than traditional wheeled vehicles. Nonetheless, Skytran will be quite speedy as its vehicles will be able to travel at a velocity of 100 miles per hour [9].

The power needed for avoiding contact with the tracks will be just a paltry fraction of the entire system energy consumption which will be very fuel economy - over 200 miles per US gallon [4]. Most of the energy will be consume to overcome drag as other Maglev transportation systems.

Maglev systems typically have lower maintenance costs; however, usually their construction costs are much higher. The first generation of Maglev transportation systems has been implemented in just three countries - China [10], Japan [11] and South Korea [12]. SkyTran is planned to be implemented in several countries. The first country is Israel [13], but there are also blueprints for France and India [14]. The United States also expressed interest in the SkyTran project [15].

If a damage generated by any incident like erosion, attrition or wearing down befalls, an alert should be immediately sent to the control center in order to stop the traffic in this segment. In such circumstances time is very critical because the alert should be sent before any vehicle enters the damage segment so as to avoid a possible crash. An automatic scheduler [16] will direct the vehicles via its communication system [17] to skirt the damaged segment.

A conventional digital camera can be helpful to locate damaged tracks. JPEG is the most popular format that digital cameras generate. Actually, JPEG is commonly employed by most of electronic imaging equipment like scanners or digital cameras [18] together with vehicle devices like GPS [19] in addition to damage locator systems like Fuselage Damage Locator [20, 21].

JPEG is the most popular technique for image compression and has various advantages like the capability of being decompressed in parallel [22], the uncomplicatedness of adaptation for hardware implementation [23] and the simplicity of interfacing with other algorithms $[24,25]$. 
Usually images are compressed before they are sent to the disk. Sometimes it could be unwise to decompress the image and just when we have the original data to execute the required process on this original image. As a better alternative, sometimes it can be better to process the original image. Such a processing will be faster. In addition, JPEG works on a frequency space and the data of this space can be very useful as we will show in the next section and besides we will not have to adjust the conventional digital camera to fit our system, but rather we will be able to use the digital camera as it is.

The rest of the paper is organized as follow: Section 2 explains how the data of JPEG format can be employed for detecting damaged tracks; Section 3 describes the evaluation of our proposed technique while section 4 concludes the paper.

\section{Automatic Persistent Inspection of SkyTran Track}

JPEG format is the most common format for image compression [26]. Almost all image compression formats lose information and JPEG is no exception. When someone decodes an image that has been compressed in such a technique, he will get a very similar image with unnoticeable changes; though not exactly the same original image. The quality of the compressed images can be decided by the user; however, most of the users prefer the defaults of their devices which typically give much better images than a human eye in fact needs. JPEG is in the mark for about quarter of century and is described in numerous papers e.g., [27, 28, 29].

The first phase of JPEG format is executing DCT [30]. DCT converts the image into a frequency space. In the second phase, JPEG divides the frequency coefficients and rounds them to the closest integer. These coefficients are typically of very low magnitude, therefore they are rounded to zero. If most of the coefficients in a JPEG block are zero or of very low magnitude, JPEG will produce a very short bits sequence representing this block, because JPEG handles zero sequences very efficiently.

If there is a sharp change in a JPEG block, DCT will produce high value for many frequency coefficients. High values will be compressed into much longer sequence bits.

There is a big difference between an image of smooth tracks and an image containing tracks with damages. Accordingly, we can use the blocks of JPEGS and look in the compressed blocks for long bit sequences. If there are blocks which have been compressed into long bit sequences, they will be suspected for being blocks of damaged tracks.

In our application we took a straightforward technique. We take pictures of the tracks. In point of fact we take a close picture of every track segment. If the entire size of one image is above a certain threshold, we will suspect this track segment as a damaged segment.

When there is uncertainty about the threshold value, probability density function (PDF) of the block can be analyzed so as to decide on the most appropriate value. If the image is simple, the PDF will be mono-modal and the inflection point value can be set as the threshold value $[31,32,33]$.

Figure 3 shows how JPEG format can be employed for detecting sharp changes. The image in Figure 2 has been compressed in grayscale baseline JPEG format using quality of $75 \%$ in size of $1200 \times 1200$ pixels. 


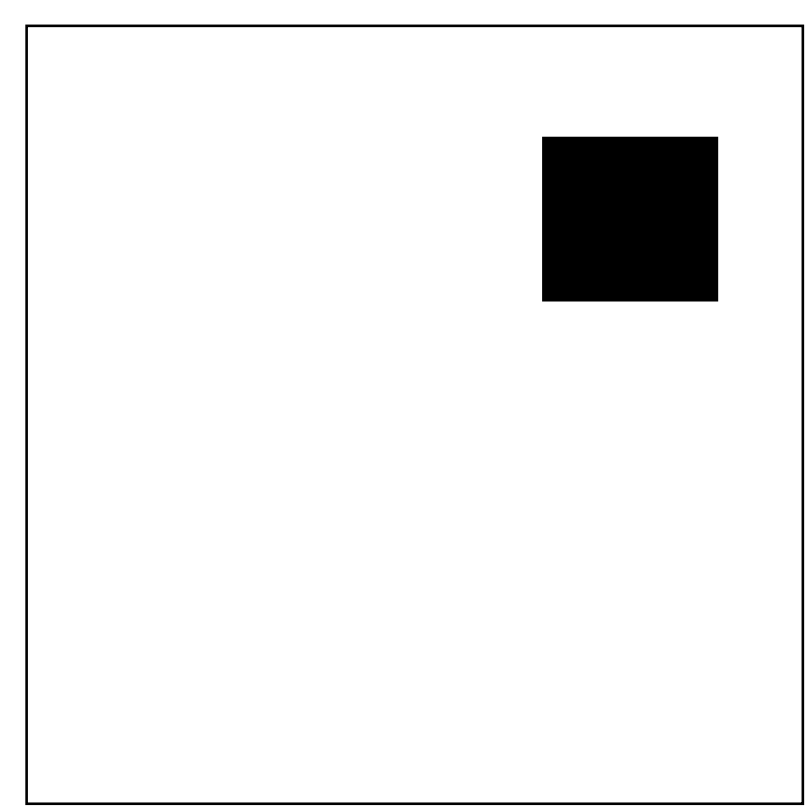

Figure 3. Sample Image and How JPEG can be Used for Contouring

JPEG format saves the difference between the previous block DC coefficient and the current block DC coefficient. If the block is within a smooth area, there will be no changes between the DC coefficients. Such blocks are encoded as six bits by JPEG format:

$$
0,0,1,0,1,0 \text {. }
$$

The 0,0 indicate that there is no difference between the DC coefficients of the previous and the current block. 1,0,1,0 indicate the end of the block. When there is a difference between the values of the DC coefficients of the previous and the current blocks, the size of the encoded block will be slightly larger.

Table 1. JPEG Format of the Upper Left Corner of the Black Square

\begin{tabular}{|c|c|c|c|c|c|c|c|}
\hline-12 & 18 & -8 & -2 & 3 & -1 & -1 & 1 \\
\hline 22 & -25 & 8 & 2 & -3 & 0 & 1 & -1 \\
\hline-18 & 20 & -7 & -2 & 2 & 0 & -1 & 1 \\
\hline 16 & 14 & 4 & 1 & -1 & 0 & 1 & 1 \\
\hline-11 & 10 & -2 & -1 & 0 & -1 & -1 & 0 \\
\hline 6 & -5 & 1 & -3 & 0 & 0 & 0 & 0 \\
\hline-2 & 2 & -1 & 0 & 0 & 0 & 0 & 0 \\
\hline 1 & -1 & 0 & 0 & 0 & 0 & 0 & 0 \\
\hline
\end{tabular}


Yet, the DC values are the minor part of the compressed block. Table 1 shows the compressed block of the upper left corner of the black square. The size of the black square is 240X240 pixels and the square is not aligned to JPEG blocks. Such data which have many non-zero coefficients is compressed by JPEG as many more bits.

A block which encodes a sharp change from white to black is represented by a wide range of non-zero frequency coefficients. Figure 2 and Table 1 show a sample of such a block, which contains the upper left corner of the black square. Compressing these values in JPEG format will require 243. The difference between 6 to 243 is clear and obvious, so it is easy to select a threshold that detects only images of damage tracks [34, 35, 36].

\section{Evaluation}

JPEG has a quality factor that is employed for deciding on two quantization tables one table for the luminance (brightness) information and another table for the chrominance (color) information. Many applications support IJG (Independent JPEG Group). These applications employ quantization tables according to the IJG specification; however, it should be noted that there are many other applications employing different quantization tables. In this paper we have used IJG standard quantization tables. Additionally, in our system, we obviously preferred the highest quality factor (100\%), so as to obtain the best distinguishableness. In IJG standard (and most of the other standards) it means that the entire quantization table is filled with 1s i.e. the frequency coefficients are not divided; however, they are still rounded to the closest integer.

By using the size of the images we actually calculate the average size of its blocks and according to this value we can have an idea which track segments are damaged [37, 38].

We checked our technique on some damaged tracks in an attempt to check whether we succeed to locate the damaged segment. Undesirably, obvious cases like in Figure 2 do not exist in real track segments; however we still succeed to find most of the damaged segments by analyzing their images like in Figure 4 which shows a track with a large scratch in the bottom.

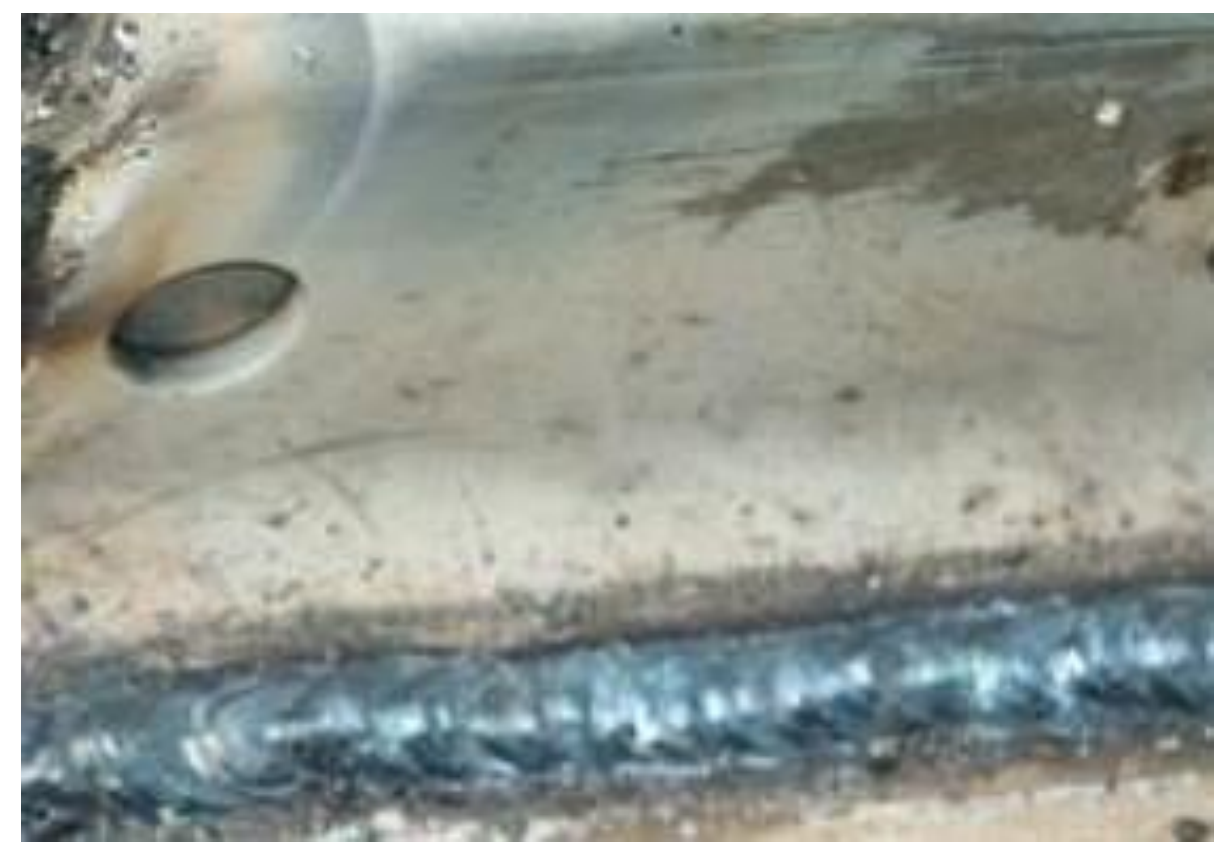

Figure 4. Damaged Track

We have used Nikon D5300 digital camera [39] with resolution of 24.2 MP. Images that have been compressed into more than $4.9 \mathrm{MB}$ typically indicated a damaged track 
segment; whereas images that have been compressed into less than 3.7MB typically indicated an in order track segment. The sizes between $3.7 \mathrm{MB}$ to $4.9 \mathrm{MB}$ were uncertain.

There have been also cases where our system has unfortunately created false alarms. Figure 5 shows a case of such a false alarm. The white dirt on the track in Figure 5 can be clearly noticed. This dirt caused a false alarm as this track segment is damaged; whereas this track segment is actually in order.

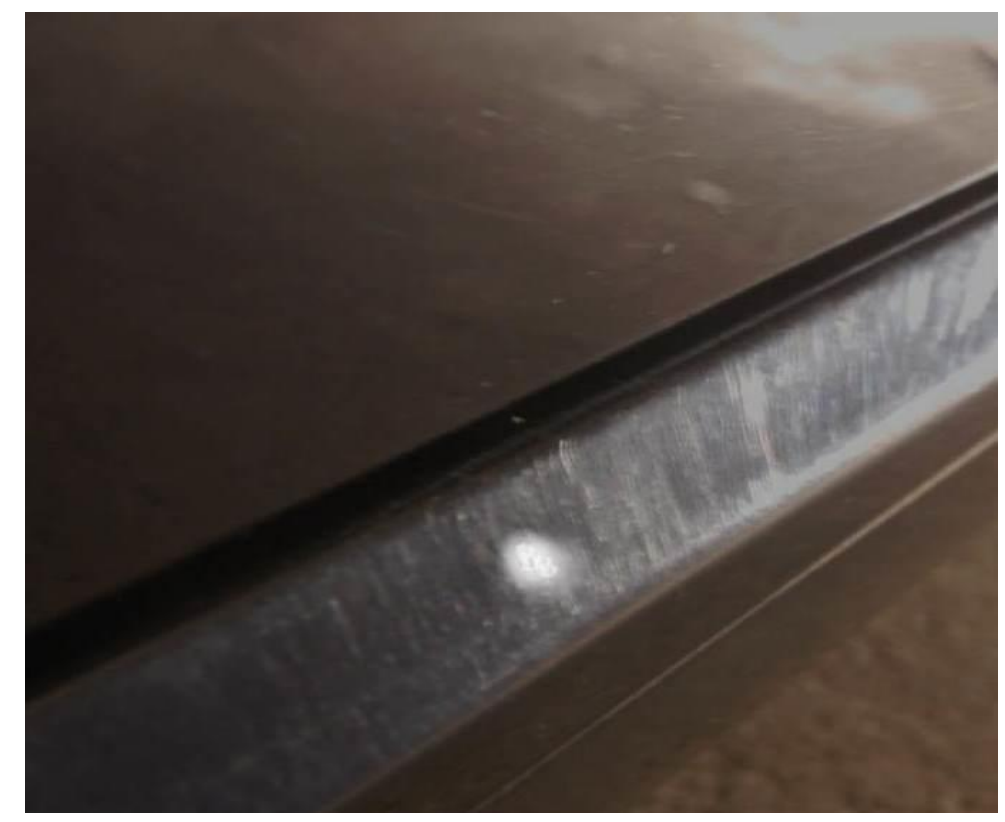

Figure 5. Dirt on the Tracks

Bad weather can also produce false alarm circumstances. Snow or hail can fall on the tracks and make their color uneven. An example of a close look at such tracks can be seen in Figure 6. The system can mistakenly consider that snow or hail as damage and can produce a false alarm.

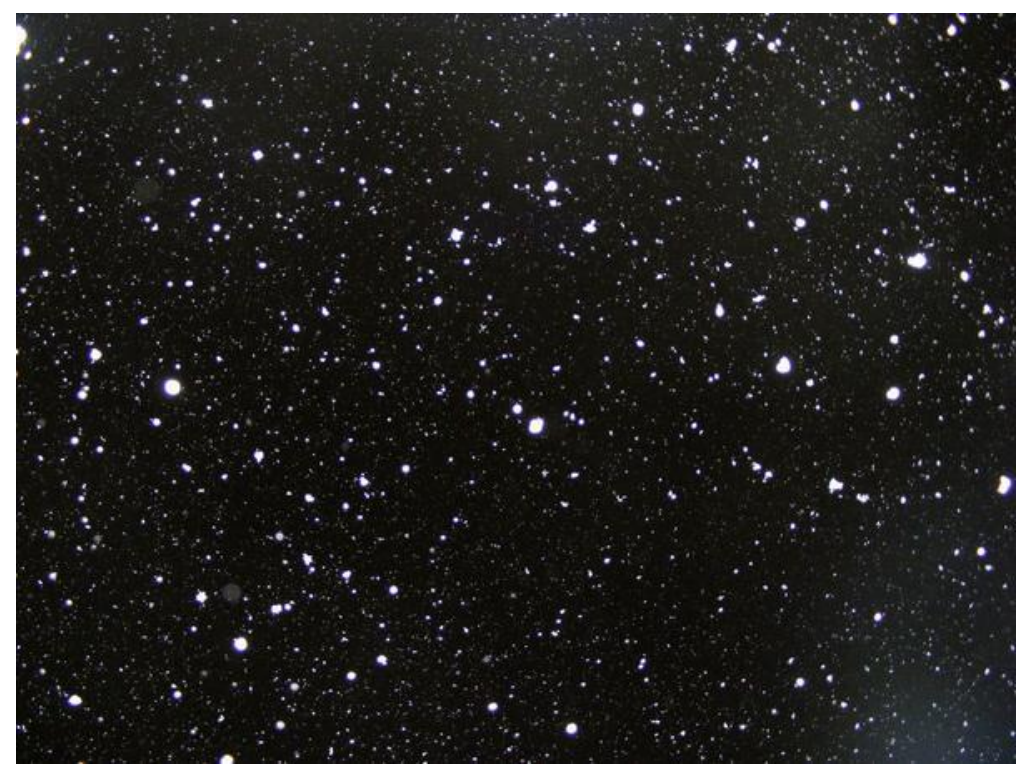

Figure 6. Small Snowflakes on a Track 


\section{Conclusions}

This paper has suggested employing of a rudimentary digital camera that is able to produce JPEG images for detecting damaged SkyTran segments. The digital camera persistently takes pictures of all the SkyTran track system. JPEG assumes that all of images are pretty smooth. Usually this assumption is correct; however, in any JPEG individual block, when there is a sharp change in the block, the value of many of its AC frequency coefficients will be non-zero; therefore the block will be compressed into a longer sequence of bits. For that reason, if many blocks are large, the entire image will be very large.

Devices for tracking down such large images can be mounted on the SkyTran track system. If a damaged segment is detected, the vehicles will not go through this segment; therefore these devices expectantly will save lives.

\section{References}

[1] S. Pravas, "SkyTran-Personal Rapid Transit", Engineers Garage Knowledge Base, https://www.engineersgarage.com/articles/skytran-personal-rapid-transit, (2016).

[2] K. Sossoe and J. P. Lebacque, "Dynamic Model for Assignment in a 'Sky-Car'Transit System: Spatial Interactions with Other Common Transport Modes", Traffic and Granular Flow, pp. 499-506, Springer International Publishing, (2016).

[3] H. W. Lee, K. C. Kim and J. Lee, "Review of maglev train technologies", IEEE transactions on magnetics, vol. 42, no. 7, (2006), pp. 1917-1925.

[4] Y. Wiseman, "Automatic Persistent Inspection of SkyTran Track System", http://u.cs.biu.ac.il/ wiseman/skytran1.pdf , (2017).

[5] W. Zhao, M. Cheng, J. Ji, R. Cao, Y. Du and F. Li, "Design and analysis of a new fault-tolerant linear permanent-magnet motor for maglev transportation applications", IEEE Transactions on Applied Superconductivity, vol. 22, no. 3, (2012).

[6] X. Chen, F. Tang, Z. Huang and G. Wang. "High-speed maglev noise impacts on residents: A case study in Shanghai", Transportation Research Part D: Transport and Environment, vol. 12, no. 6, (2007), pp. 437-448.

[7] P. J. Lee and M. J. Griffin, "Combined effect of noise and vibration produced by high-speed trains on annoyance in buildings", The Journal of the Acoustical Society of America, Vol. 133, no. 4, (2013), pp. 2126-2135.

[8] M. Yadav, N. Mehta, A. Gupta, A. Chaudhary and D. V. Mahindru, "Review of magnetic levitation (MAGLEV): A technology to propel vehicles with magnets." Global Journal of Research in Engineering, vol. 13, no. 7, (2013).

[9] D. J. Malewicki, "Silicon Is About to Change the World-Again", Proceedings of the IEEE, Vol. 97, no. 11, (2009), pp. 1750-1753.

[10] Q. Yuan, G. Long, Z. Liu, K. Ma, Y Xie, D. Deng and H. Huang, "Sealed-space-filling SCC: A special SCC applied in high-speed rail of China", Construction and Building Materials, vol. 124, (2016), pp. 167-176

[11] M. Uno, "Chuo Shinkansen Project using Superconducting Maglev System", Japan Railway \& Transport Review, vol. 68, (2016), pp. 14-25.

[12] K. B. Lee and J. C. Kim, "A Study on Energy Efficiency Analysis by Changing Maglev Train Slip Frequency", International Journal of Applied Engineering Research, vol. 11, no. 2, (2016) , pp. 11091112.

[13] Israel Aerospace Industries (IAI) News, "Skytran and iai to Build a Demonstration System for SkyTran Magnetic Levitation Transportation System", http://www.iai.co.il/2013/32981-46034en/MediaRoom_News.aspx, (2014).

[14] SkyTran, "SkyTran - About", http://www.skytran.com/skytran/, (2017).

[15] P. Kavilanz, "Sky taxis are about to become a reality", CNN Money, http://money.cnn.com/2015/10/23/smallbusiness/skytran-air-taxis/, (2015).

[16] R. Ben Yehuda and Y. Wiseman, "The Offline Scheduler for Embedded Transportation Systems", Proceedings of IEEE Conference on Industrial Electronics (IEEE ICIT-2011), Auburn, Alabama, (2011), pp. 449-454.

[17] Y. Wiseman, K. Schwan and P. Widener, "Efficient End to End Data Exchange Using Configurable Compression", Operating Systems Review, ACM Special Interest Group on Operating Systems (SIGOPS), vol. 39, no. 3, (2005), pp. 4-23.

[18] N. Khanna, G. T. C. Chiu,, J. P. Allebach and E.J. Delp, "Forensic techniques for classifying scanner, computer generated and digital camera images", Proceedings of the 2008 IEEE International Conference 
on Acoustics, Speech, and Signal Processing (ICASSP 2008), Las Vegas, Nevada, USA, (2008), pp. 1653-1656.

[19] P. Hongyan, H. Hong, J. Hengtian, "Drive design for ship GPS navigation equipment based on Linux operating system", International Conference on Educational and Network Technology (ICENT), Qinhuangdao, China, June, (2010), pp. 384-388.

[20] Y. Wiseman, "Device for Detection of Fuselage Defective Parts", Information Journal, Tokyo, Japan, vol. 17(9(A)), (2014), pp. 4189-4194.

[21] Y. Wiseman, "Fuselage Damage Locator System, Advanced Science and Technology Letters, vol. 37, (2013), pp. 1-4.

[22] S. T. Klein and Y. Wiseman, "Parallel Huffman Decoding with Applications to JPEG Files", The Computer Journal, Oxford University Press, Swindon, United Kingdom, vol. 46, no. 5, (2003), pp. 487497.

[23] Y. Wiseman, "A Pipeline Chip for Quasi Arithmetic Coding", IEICE Journal - Trans. Fundamentals, Tokyo, Japan, Vol. E84-A, no.4, (2001), pp. 1034-1041.

[24] Y. Wiseman, "Burrows-Wheeler Based JPEG", Data Science Journal, vol. 6, (2007), pp. 19-27.

[25] Klein S. T. and Y. Wiseman, "Parallel Lempel Ziv Coding", Journal of Discrete Applied Mathematics, vol. 146, no. 2, (2005), pp. 180-191.

[26] J. Fridrich, M. Goljan and D. Hogea, "Steganalysis of JPEG images: Breaking the F5 algorithm", International Workshop on Information Hiding, Noordwijkerhout, The Netherlands, Published by Springer, (2002), pp. 310-323.

[27] Information Technology - Digital Compression and Coding of Continuous-Tone Still Images Requirements and Guidelines - International Standard ISO/IEC 10918-1, (1993).

[28] G. K. Wallace, "The JPEG Still Picture Compression Standard", Communication of the ACM, vol. 34, (1991), pp. 3-44.

[29] Y. Wiseman, "The still image lossy compression standard - JPEG", Encyclopedia of Information and Science Technology, Third Edition, Published by IGI-Global, vol. 1, Chapter 28, (2014), pp. 295-305.

[30] V. Britanak and P. C. Yip and K. R. Rao, "Discrete cosine and sine transforms: general properties, fast algorithms and integer approximations", Elsevier, (2010).

[31] Y. Wiseman, "Tool for Online Observing of Traffic Congestions", International Journal of Control and Automation, vol. 10, no. 6, (2017).

[32] Y. Wiseman, "Computerized Traffic Congestion Detection System", International Journal of Transportation and Logistics Management, vol. 1, no. 1, (2017), pp. 1-8.

[33] Y. Wiseman "Real-Time Monitoring of Traffic Congestions", IEEE International Conference on Electro Information Technology (EIT 2017), Lincoln, Nebraska, USA, (2017).

[34] Y. Wiseman, "Take a Picture of Your Tire!", Proc. IEEE Conference on Vehicular Electronics and Safety (IEEE ICVES-2010) Qingdao, ShanDong, China, (2010), pp. 151-156.

[35] Y. Wiseman, "The Effectiveness of JPEG Images Produced By a Standard Digital Camera to Detect Damaged Tyres", World Review of Intermodal Transportation Research, vol. 4, no. 1, (2013), pp. 23-36.

[36] Y. Wiseman, "Camera That Takes Pictures of Aircraft and Ground Vehicle Tires Can Save Lives", Journal of Electronic Imaging, vol. 22, no. 4, (2013).

[37] Y. Wiseman and E. Fredj, "Contour Extraction of Compressed JPEG Images", ACM - Journal of Graphic Tools, vol. 6 no. 3, pp. 37-43, (2001).

[38] E. Fredj and Y. Wiseman, "An O(n) Algorithm for Edge Detection in Photos Compressed by JPEG Format", Proc. IASTED International Conference on Signal and Image Processing SIP-2001, Honolulu, Hawaii, (2001), pp. 304-308.

[39] S. Watanabe, "Digital camera", U.S. Patent D749,658, issued February 16, (2016).

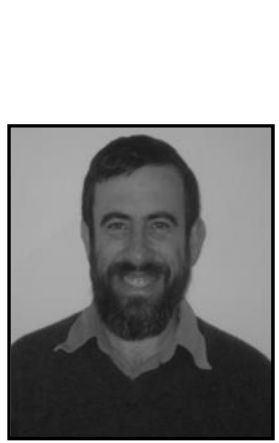

\section{Author}

Yair Wiseman, He got a Summa Cum Laude M.Sc. and a PhD from Bar-Ilan University and completed two Post-Doc - one at the Hebrew University of Jerusalem and one in Georgia Institute of Technology.

Dr. Wiseman's research interests include Computational Transportation Science, Intelligent Transportation Systems, Process Scheduling, Hardware-Software Codesign, Memory Management, Computer Clusters, Data Compression, JPEG, Embedded Systems, Real-Time Systems and Operating Systems.

Dr. Wiseman is on the editorial board of several journals, a member of dozens of conference committees and a reviewer of many scholarly journals. Dr. Wiseman authored two books as well. 
In addition, Dr. Wiseman has been teaching in many institutes including Bar-Ilan University, The Hebrew University of Jerusalem, Israel Aircraft Industry, Holon Institute of Technology and Jerusalem College of Technology.

Dr. Wiseman has been supervising many graduate students and an interesting point is that Albert Einstein is Dr. Wiseman's academic great-great-grandfather (i.e. the advisor of the advisor of the advisor of Dr. Wiseman's advisor).

Dr. Wiseman has collaborated with other partners and received research grants to run an active laboratory from inter alia Sun Microsystems, Intel, Polak Foundation and the Open University.

Dr. Wiseman is an international expert who has reviewed and evaluated several large projects of the European Union, Israel Science Foundation, MB Logic and more. Dr. Wiseman's papers have been published in many venues around the world. 
International Journal of Control and Automation

Vol.10, No.7 (2017) 